\title{
Recursion operator and dispersionless rational Lax representation
}

\author{
K. Zheltukhin \\ Department of Mathematics, Faculty of Sciences, Bilkent University, 06533 Ankara, Turkey \\ Received 19 September 2001; received in revised form 13 February 2002; accepted 27 March 2002 \\ Communicated by A.P. Fordy
}

\begin{abstract}
We consider equations arising from dispersionless rational Lax representations. A general method to construct recursion operators for such equations is given. Several examples are given, including a degenerate bi-Hamiltonian system with a recursion operator. () 2002 Elsevier Science B.V. All rights reserved.
\end{abstract}

PACS: 02.30.Ik; 02.30.Sr

Keywords: Integrable system; Recursion operator

\section{Introduction}

Recently a new method of constructing a recursion operator from Lax representation was introduced in [1]. This construction depends on Lax representation of a given system of PDEs. Let

$$
L_{t}=[A, L],
$$

be Lax representation of an integrable nonlinear system of PDEs. Then a hierarchy of symmetries can be given by

$$
L_{t_{n}}=\left[A_{n}, L\right], \quad n=0,1,2, \ldots,
$$

where $t_{0}=t, A_{0}=A$ and $A_{n}, n=0,1,2, \ldots$, are Gel'fand-Dikkii operators given in terms of $L$. The recursion relation between symmetries can be written as

$$
L_{t_{n+1}}=L L_{t_{n}}+\left[R_{n}, L\right], \quad n=0,1,2, \ldots,
$$

where $R_{n}$ is an operator such that ord $R_{n}=\operatorname{ord} L$.

This symmetry relation allows us to find $R_{n}$, hence $L_{t_{n+1}}$, in terms of $L$ and $L_{t_{n}}$.

In $[1,2]$ this method was applied to construct recursion operators for Lax equations with different classes of scalar and shift operators, corresponding to field and lattice systems, respectively. In [3] the method was applied to

E-mail address: zhelt@fen.bilkent.edu.tr (K. Zheltukhin). 
dispersionless Lax equations on a Poisson algebra of Laurent series

$$
\Lambda=\left\{\sum_{-\infty}^{+\infty} u_{i} p^{i}: u_{i} \text {-smooth functions }\right\}
$$

with a polynomial Lax function. The present work is a continuation of [3]. Here we consider a dispersionless Lax equation on the Poisson algebra $\Lambda$ with a rational Lax function. Such equations one can find in context of topological field theories (see [4,5]).

We have a Lax function

$$
L=\frac{\Delta_{1}}{\Delta_{2}}
$$

where $\Delta_{1}, \Delta_{2}$ are polynomials of degree $N$ and $M$, respectively, and $N>M$. The dispersionless Lax equation is

$$
\frac{\partial L}{\partial t_{n}}=\left\{\left(L^{\frac{1}{N-M}+n}\right)_{\geqslant 0}, L\right\}, \quad n=0,1,2, \ldots
$$

where the Poisson bracket is given by

$$
\{f, g\}=p\left(\frac{\partial f}{\partial p} \frac{\partial g}{\partial x}-\frac{\partial f}{\partial x} \frac{\partial g}{\partial p}\right) .
$$

Eq. (6) is of hydrodynamic type. There are several methods for construction of a recursion operator for some equations of hydrodynamic type (see [6-8]). Also a recursion operator can be found with the help of two compatible Hamiltonian formulations of a given equation. For Hamiltonian formulations of equations of hydrodynamic type see Refs. $[9,10]$ and for Hamiltonian formulations of the equations admitting a dispersionless Lax representation see Refs. [11-15].

We construct a recursion operator for a hierarchy of symmetries (6), using a dispersionless Lax representation. First we study the symmetry relation (3) for the rational Lax function. Then we give some examples of calculation of a recursion operator. In particular, we find a recursion operator $\mathcal{R}$ for Eq. (6) with the Lax function

$$
L=p+S+\frac{P}{p+Q},
$$

which leads to the system [11]

$$
S_{t}=P_{x}, \quad P_{t}=P S_{x}-Q P_{x}-P Q_{x}, \quad Q_{t}=Q S_{x}-Q Q_{x} .
$$

The recursion operator is given by

$$
\mathcal{R}=\left(\begin{array}{ccc}
S & 1 & P Q^{-1}+P_{x} D_{x}^{-1} \cdot Q \\
2 P & S-Q & -2 P+\left(P S_{x}-(P Q)_{x}\right) D_{x}^{-1} \cdot Q \\
Q & 1 & P Q^{-1}+S-Q+\left(Q S_{x}-Q Q_{x}\right) D_{x}^{-1} \cdot Q
\end{array}\right) .
$$

In [11] bi-Hamiltonian representation of this equation was constructed with Hamiltonian operators

$$
\mathcal{D}_{1}=\left(\begin{array}{ccc}
0 & P & Q \\
P & -2 P Q & -Q^{2} \\
Q & -Q^{2} & 0
\end{array}\right) D_{x}+\left(\begin{array}{ccc}
0 & P_{x} & Q_{x} \\
0 & -(P Q)_{x} & -Q Q_{x} \\
0 & -Q Q_{x} & 0
\end{array}\right),
$$

and

$$
\mathcal{D}_{2}=\left(\begin{array}{ccc}
2 P & P(S-3 Q) & Q(S-Q) \\
P(S-3 Q) & P\left(2 P-2 S Q+4 Q^{2}\right) & Q\left(2 P-S Q+Q^{2}\right) \\
Q(S-Q) & Q\left(2 P-S Q+Q^{2}\right) & 2 Q^{2}
\end{array}\right) D_{x}
$$




$$
+\left(\begin{array}{ccc}
P_{x} & S P_{x}-2(P Q)_{x} & S Q_{x}-Q Q_{x} \\
P S_{x}-(Q P)_{x} & \left(-S P Q+P^{2}+2 P Q^{2}\right)_{x} & Q_{x}\left(2 P+Q^{2}-S Q\right) \\
Q S_{x}-Q Q_{x} & Q\left(2 P_{x}+2 Q Q_{x}-S_{x}-S Q Q_{x}\right) & 2 Q Q_{x}
\end{array}\right) .
$$

These Hamiltonian operators are degenerate, so, one cannot use them to find a recursion operator. But it turns out that they are related to the recursion operator $\mathcal{R}$. One can easily check that the following equality holds

$$
\mathcal{R} \mathcal{D}_{1}=\mathcal{D}_{2} \text {. }
$$

We observe that the degeneracy in the bi-Hamiltonian operators is due to the following fact. Let $p^{\prime}=p+F$ then the Lax function becomes

$$
L=p^{\prime}+G+\frac{P}{p^{\prime}} .
$$

This means that we have two independent variables $P$ and $G$, where $G=S-F$. The equation corresponding to the Lax function (13) has been studied in [3].

To remove degeneracy one can take the Lax function as

$$
L=p+S+\frac{P}{p}+\sum_{i=1}^{m} \frac{Q_{i}}{p+F_{i}} .
$$

As an example we shall consider the Eq. (6) with the Lax function

$$
L=p+S+\frac{P}{p}+\frac{Q}{p+F} .
$$

\section{Symmetry relation for rational dispersionless Lax representation}

Following [1] we consider the hierarchy of symmetries for the dispersionless Lax equation (6) with the Lax function (5)

$$
\frac{\partial L}{\partial t_{n}}=\left\{\left(L^{\frac{1}{N-M}+n}\right)_{\geqslant 0}, L\right\}, \quad n=0,1,2, \ldots,
$$

Lemma 1. For any $n=0,1,2, \ldots$,

$$
\frac{\partial L}{\partial t_{n}}=L \frac{\partial L}{\partial t_{n-1}}+\left\{R_{n}, L\right\} .
$$

Function $R_{n}$ has a form

$$
R_{n}=A+\frac{B}{\Delta_{2}},
$$

where $A$ is a polynomial of degree $(N-M)$ and $B$ is a polynomial of degree $(M-1)$.

Proof. We have

$$
\left(L^{\frac{1}{N-M}+n}\right)_{\geqslant 0}=\left[L\left(L^{\frac{1}{N-M}+(n-1)}\right)_{\geqslant 0}+L\left(L^{\frac{1}{N-M}+(n-1)}\right)_{<0}\right]_{\geqslant 0} .
$$

So,

$$
\left(L^{\frac{1}{N-M}+n}\right)_{\geqslant 0}=L\left(L^{\frac{1}{N-M}+(n-1)}\right)_{\geqslant 0}+\left(L\left(L^{\frac{1}{N-M}+(n-1)}\right)_{<0}\right)_{\geqslant 0}-\left(L\left(L^{\frac{1}{N-M}+(n-1)}\right)_{\geqslant 0}\right)_{<0} .
$$


If we take

$$
R_{n}=\left(L\left(L^{\frac{1}{N-M}+(n-1)}\right)_{<0}\right)_{\geqslant 0}-\left(L\left(L^{\frac{1}{N-M}+(n-1)}\right)_{\geqslant 0}\right)_{<0}
$$

then

$$
\left(L^{\frac{1}{N-M}+n}\right)_{\geqslant 0}=L\left(L^{\frac{1}{N-M}+(n-1)}\right)_{\geqslant 0}+R_{n} .
$$

Hence,

$$
\frac{\partial L}{\partial t_{n}}=\left\{\left(L^{\frac{1}{N-M}+n}\right)_{\geqslant 0}, L\right\}=\left\{L\left(L^{\frac{1}{N-M}+(n-1)}\right)_{\geqslant 0}+R_{n}, L\right\}=L \frac{\partial L}{\partial t_{n}}+\left\{R_{n}, L\right\},
$$

and (17) is satisfied. The remainder $R_{n}$ has form (18). Indeed in (19) we set

$$
A=\left(L\left(L^{\frac{1}{N-M}+(n-1)}\right)_{<0}\right)_{\geqslant 0},
$$

and

$$
B=\Delta_{2} \cdot\left(L\left(L^{\frac{1}{N-M}+(n-1)}\right)_{\geqslant 0}\right)_{<0} .
$$

Then $A$ is a polynomial of degree $(N-M-1)$ and $B$ is a polynomial of degree $(M-1)$.

Now we can apply the Lemma 1 to find recursion operators.

\section{Examples}

Example 2. Let us consider the Eq. (8) given in introduction.

Lemma 3. A recursion operator for (8) is given by (9).

Proof. Using (18) for $R_{n}$, we have $R_{n}=A+\frac{B}{p+Q}$. So, the symmetry relation (17) is

$$
\begin{aligned}
\frac{\partial S}{\partial t_{n}}+ & \frac{\partial P}{\partial t_{n}} \cdot \frac{1}{p+Q}+\frac{\partial Q}{\partial t_{n}} \cdot \frac{P}{(p+Q)^{2}} \\
= & \left(p+S+\frac{P}{p+Q}\right)\left(\frac{\partial S}{\partial t_{n-1}}+\frac{\partial P}{\partial t_{n-1}} \cdot \frac{1}{p+Q}+\frac{\partial Q}{\partial t_{n-1}} \cdot \frac{P}{(p+Q)^{2}}\right) \\
& +p\left(A_{x}+\frac{B_{x}}{p+Q}+\frac{-B Q_{x}}{(p+Q)^{2}}\right)\left(1+\frac{-P}{(p+Q)^{2}}\right)-\frac{p B}{(p+Q)^{2}}\left(S_{x}+\frac{P_{x}}{p+Q}+\frac{-P Q_{x}}{(p+Q)^{2}}\right) .
\end{aligned}
$$

To have the equality the coefficients of $p$ and $(p+Q)^{-3}$ must be zero. It gives the recursion relations to find $A$ and $B$. Then the coefficients of $p^{0},(p+Q)^{-1},(p+Q)^{-2}$ give expressions for $\frac{\partial S}{\partial t_{n}}, \frac{\partial P}{\partial t_{n}}, \frac{\partial Q}{\partial t_{n}}$.

Example 4. The dispersionless Lax equation (6) with the Lax function (15), for $n=1$, gives the following system

$$
S_{t}=P_{x}+Q_{x}, \quad P_{t}=P S_{x}, \quad Q_{t}=Q S_{x}-F Q_{x}-Q F_{x}, \quad F_{t}=F S_{x}-F F_{x} .
$$


Lemma 5. A recursion operator for (20) is given by

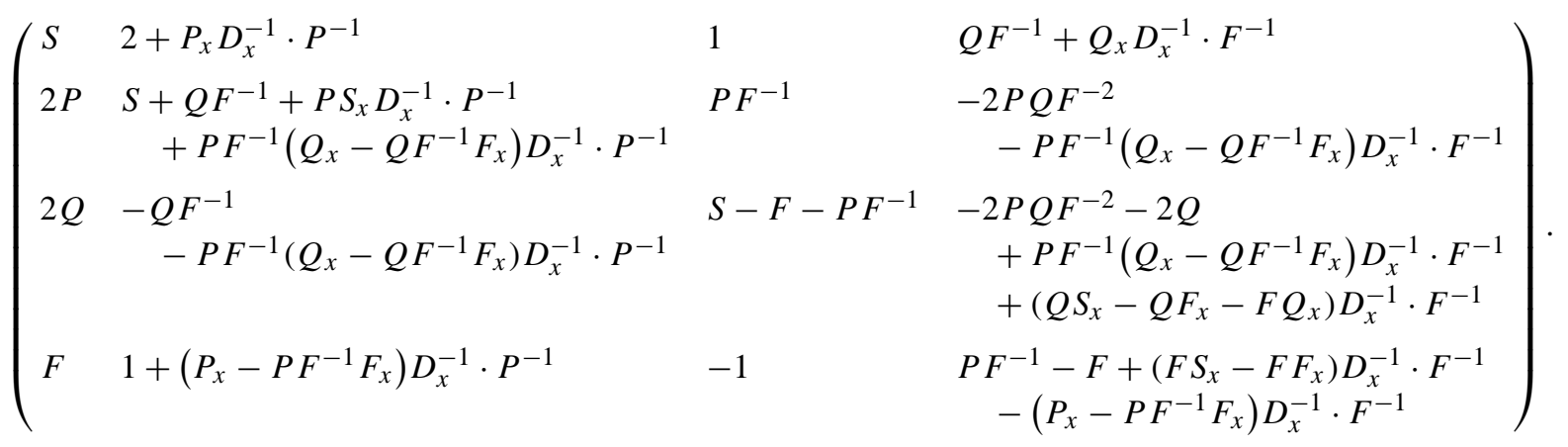

Proof. Using (18) for $R_{n}$, we have $R_{n}=C+\frac{A}{p}+\frac{B}{p+F}$. So, the symmetry relation (17) is

$$
\begin{aligned}
\frac{\partial S}{\partial t_{n}}+ & \frac{\partial P}{\partial t_{n}} \cdot \frac{1}{p}+\frac{\partial Q}{\partial t_{n}} \cdot \frac{1}{(p+F)}+\frac{\partial F}{\partial t_{n}} \cdot \frac{-Q}{(p+F)^{2}} \\
= & \left(p+S+\frac{P}{p}+\frac{Q}{p+F}\right)\left(\frac{\partial S}{\partial t_{n-1}}+\frac{\partial P}{\partial t_{n-1}} \cdot \frac{1}{p}+\frac{\partial Q}{\partial t_{n-1}} \cdot \frac{1}{(p+F)}+\frac{\partial F}{\partial t_{n-1}} \cdot \frac{-Q}{(p+F)^{2}}\right) \\
& +p\left(\frac{-B}{p^{2}}+\frac{-C}{(p+F)^{2}}\right)\left(S_{x}+\frac{P_{x}}{p}+\frac{Q_{x}}{(p+F)}+\frac{-Q F_{x}}{(p+F)^{2}}\right) \\
& -p\left(A_{x}+\frac{B_{x}}{p}+\frac{C_{x}}{(p+F)+\frac{-C F_{x}}{(p+F)^{2}}}\right)\left(1+\frac{P}{p}+\frac{-Q}{(p+F)^{2}}\right) .
\end{aligned}
$$

Therefore, the coefficients of $p, p^{-2}$ and $(p+F)^{-3}$ must be zero, it gives recursion relations to find $A, B$ and $C$. Then the coefficients of $p^{0}, p^{-1},(p+F)^{-1}$ and $(p+F)^{-2}$, give expressions for $\frac{\partial S}{\partial t_{n}}, \frac{\partial P}{\partial t_{n}}, \frac{\partial Q}{\partial t_{n}}$ and $\frac{\partial F}{\partial t_{n}}$.

\section{Acknowledgements}

I thank Professors Metin Gürses, Atalay Karasu and Maxim Pavlov for several discussions. This work is partially supported by the Scientific and Technical Research Council of Turkey.

\section{References}

[1] M. Gürses, A. Karasu, V.V. Sokolov, J. Math. Phys. 40 (1999) 6473.

[2] M. Blaszak, Rep. Math. Phys. 48 (1-2) (2001) 27.

[3] M. Gürses, K. Zheltukhin, J. Math. Phys. 42 (2001) 1309.

[4] B.A. Dubrovin, Geometry of 2D Topological Field Theories, Lecture Notes in Mathematics, Vol. 1620, Springer, Berlin, 1993, pp. 120348.

[5] S. Aoyama, Y. Kodama, Commun. Math. Phys. 182 (1996) 185.

[6] M.B. Sheftel, Generalized hydrodynamic-type systems, in: N.H. Ibragimov (Ed.), CRC Handbook of Lie Group Analysis of Differential Equations, Vol. 3, CRC Press, New York, 1996, pp. 169-189.

[7] V.M. Teshukov, LIIAN 106 (1989) 25.

[8] A.P. Fordy, B. Gürel, Theoret. Math. Phys. (1999).

[9] B.A. Dubrovin, S.P. Novikov, Sov. Math. Dokl. 27 (1983) 665. 
[10] E.V. Ferepantov, Hydrodynamic-type systems, in: N.H. Ibragimov (Ed.), CRC Handbook of Lie Group Analysis of Differential Equations, Vol. 1, CRC Press, New York, 1994, pp. 303-331.

[11] I.A.B. Strachan, J. Math. Phys. 40 (1999) 5058.

[12] D.B. Fairlie, I.A.B. Strachan, Inverse Problems 12 (1998) 885.

[13] J.C. Brunelli, M. Gürses, K. Zheltukhin, Rev. Math. Phys. 13 (4) (2001) 529.

[14] J.C. Brunelli, A. Das, Phys. Lett. A 235 (1997) 597.

[15] L.-C. Li, Commun. Math. Phys. 203 (1999) 573. 